Review

\title{
New antimicrobial approaches to gram positive respiratory infections
}

Q4 Adamantia Liapikou ${ }^{\text {a, }}{ }^{*}$, Catia Cilloniz ${ }^{\text {b }}$, Josep Mensa ${ }^{c}$, Antonio Torres ${ }^{\text {b }}$

a 6th Respiratory Department, Sotiria Chest Diseases Hospital, Athens, Greece

${ }^{\mathrm{b}}$ Department of Pneumology, Institut Clinic del Tórax, Hospital Clinic, University of Barcelona, Spain

${ }^{\mathrm{c}}$ Department of Infectious Diseases, Hospital Clinic, Spain

\section{A R T I C L E I N F O}

\section{Article history:}

Received 9 April 2014

Received in revised form

1 May 2014

Accepted 5 May 2014

Available online $\mathrm{xxx}$

\section{Q5 Keywords:}

Gram (+) respiratory pathogens

MRSA

Antibiotic resistance

New antibiotics

Treatment

Chemical compounds studied in this article:

Ceftaroline fosamil (Pubmed CID:73425380)

Telavancin (Pubmed CID:3081362)

Cethromycin (Pubmed CID: 447451)

Solithromycin (Pubmed CID: 56842140)

Omadacycline (Pubmed CID:54697325)

Ceftopibrole (Pubmed CID: 6918430)

Nemonoxacin (Pubmed CID: 11993740)

Tedizolid phosphate (Pubmed CID:

11476460)

\begin{abstract}
A B S T R A C T
Nowadays, we face growing resistance among gram-positive and gram-negative pathogens that cause respiratory infection in the hospital and in the community. The spread of penicillin- and macrolideresistant pneumococci, Community-acquired methicillin-resistant staphylococcus aureus (Ca-MRSA), the emergence of glycopeptide-resistant staphylococci underline the need for underline the need for therapeutic alternatives. A number of new therapeutic agents, with activity against the above Gram $(+)$ respiratory pathogens, as ceftaroline, ceftopibrole, telavancin, tedizolid have become available, either in clinical trials or have been approved for clinical use. Especially, the development of new oral antibiotics, as nemonaxacin, omadacyclin, cethromycin and solithromycin will give a solution to the lack of oral drugs for outpatient treatment. In the future the clinician needs to optimize the use of old and new antibiotics to treat gram $(+)$ respiratory serious infections.
\end{abstract}

(C) 2014 Elsevier Ltd. All rights reserved.

\section{Introduction}

Lower respiratory tract infections (LRTIs) as acute exacerbations of chronic bronchitis, community-acquired pneumonia (CAP) and hospital acquired pneumonia (HAP) are one of the most common diseases in humans and a long-term global public health concern.

Within Europe, CAP is the leading cause of death due to infection [1] with approximately $90 \%$ of deaths due to pneumonia occurring in people aged $>65$ years. Ventilator-associated pneumonia (VAP),representing $80 \%$ of HAP, is reported to be the most common hospital-acquired infection among patients requiring mechanical ventilation, carrying an attributable mortality of $33-60 \%$.

\footnotetext{
* Corresponding author.

E-mail address: mliapikou@yahoo.com (A. Liapikou).
}

Streptococcus pneumoniae, Haemophilus influenzae, Moraxella catarrhalis, Staphylococcus aureus, Enterobacteriacea, Pseudomonas aeruginosa and Mycoplasma pneumoniae, Chlamydophila pneumoniae and Legionella pneumophila causing atypical pneumonias are the major pathogens implicated in respiratory tract infections.

Nowadays, multi-drug-resistant bacteria have emerged throughout the world with more than one third of the isolates are multidrug-resistant (MDR) [2], and half the deaths from clinical infection in Europe are associated with MDR bacteria [3]. The increasing problem of antibiotic resistance means that community and hospital LRTIs are becoming progressively more difficult to diagnose and treat.

In a recent review Welte et al., analyzing forty-six primary articles of CAP, S. pneumoniae was reported as the most frequently pathogen, being isolated in $38 \%$ of outpatient cases, $27 \%$ of inpatient cases, and $28 \%$ of CAP patients admitted in the ICU [4]. In the AWARE Ceftaroline Surveillance Program (2008-2010) showed 
that among 3329 isolates of S. pneumoniae in USA, this collection contained up to $21.1 \%$ penicillin-resistant strains (using CLSI criteria for penicillin [oral penicillin V]) [5].

Among the Gram-positive respiratory organisms, S. pneumoniae resistant to penicillin and macrolides and methicillin-resistant $S$. aureus (MRSA) represent the biggest therapeutic hurdles.

Rates of penicillin resistance of $S$. pneumoniae exceeding $50 \%$ occur in certain areas of the world, such as Asia, $25 \%$ in some Mediterranean countries but remain low $(<5 \%)$ in other regions, such as Finland and Sweden [6]. Worldwide, penicillin-resistant strains of pneumococci are usually also resistant to tetracycline, erythromycin and chloramphenicol. Reports from Germany, USA and other European and Asian countries showed a resistance rate of $S$ pneumoniae to macrolides that varies from $18 \%$ to $75 \%$ [7-8].

S. aureus is the predominant Gram positive pathogen in HAP and VAP. Data from the National Nosocomial Infections Surveillance system of USA suggest that in ICUs the [9] proportion of MRSA has increased to $59.5 \%-64.4 \%$. MRSA is also commonly isolated in patients with HAP in European ICUs. Koulenti et al. [10] reported that MRSA was isolated in $16 \%$ of patients with nosocomial pneumonia (21.4\% in HAP and $14.6 \%$ in VAP). Coma is the primary risk factor for VAP caused by methacillin sensitive $S$. aureus (MSSA) and risk factors for VAP caused by MRSA include corticosteroid therapy, mechanical ventilation longer than 6 days, $>25$ yrs of age, prior diagnosis of COPD, and previous use of antibiotics [11].

Another large, prospective study reporting 474 patients with VAP in Spain found that patients with MRSA VAP had significantly higher in-hospital mortality than patients with VAP caused by other microorganisms (59.5\% versus $46.8 \%$; $p$ 0.02) [12].

Evenmore, the appearance of glycopeptide non-susceptibility among staphylococci, mainly of the vancomycin-intermediate (VISA) and hetero-VISA (hVISA) varieties, makes these infections more difficult to treat [13].

Therefore, new oral and/or parenteral antimicrobial agents with activities against these Gram-positive respiratory pathogens are in demand. To improve our fight against MRSA there are new oxazolidinone (tedizolid) and the extended spectrum cephalosporins, ceftobiprole and ceftaroline treating CAP and HAP. New agents which target protein synthesis and a quinolone are in development for the treatment of moderate to severe respiratory infections: solithromycin, cethromycin and nemonoxacin.

This review is intended to raise awareness of several novel approaches to combating the emergence of Gram (+) positiveespecially MDR-respiratory bacteria which are becoming more commonplace in our hospitals and even in our community settings.

\section{Approved antimicrobials}

A number of new therapeutic agents against Gram $(+)$ respiratory pathogens have been approved for clinical use the last 3 years, Q1 including: ceftaroline, ceftobiprole and telavancin (Table 1).

\section{Ceftaroline}

Ceftaroline fosamil is a new, bactericidal, parental, extended spectrum cephalosporin (Table 2) with activity against Gram positive organisms, including S. pneumoniae, Streptococcus pyogenes, $S$. aureus (including MRSA and vancomycin-resistant $S$. aureus (VRSA) and hetero-resistant VISA (hVISA), as well as many common Gram-negative organisms, as Hemophilus influenzae and Moraxella catarrhallis.

Like other b-lactam antibiotics, prevents cell wall formation by binding to the penicillin-binding protein (PBP), especially to PBP - 2a, which confers the methicillin resistance in $S$. aureus. The $\mathrm{MIC}_{90}$ with a range of $0.25-1$ against $S$. aureus tends to be low [14] and an MIC of $\leq 1 \mu \mathrm{g} / \mathrm{mL}$ is considered susceptible.

Ceftaroline is active against S. pneumoniae, including penicillinintermediate and-resistant strains [15]. Ceftaroline exhibits potent in vitro activity against $S$. pneumoniae with $\mathrm{MIC}_{90}$ values for penicillin-susceptible, penicillin intermediate and penicillinresistant strains of $0.015 \mathrm{mg} / \mathrm{L}, 0.06 \mathrm{mg} / \mathrm{L}$ and $0.12 \mathrm{mg} / \mathrm{L}$, respectively [14].

Using single-step and multistep passages, no resistant mutants were selected with ceftaroline in staphylococci, pneumococci, or H. influenzae [16].

Ceftaroline has limited protein binding (1-19\%) and achieved good lung penetration (40\%) in a rabbit model [17]. The major route of elimination is renal excretion with an average $t^{1 / 2}$ is $2.6 \mathrm{~h}$.

Phase III clinical trials have found that ceftaroline is non-inferior to comparator therapy for the treatment of community acquired pneumonia (FOCUS 1 and 2 trials; comparator: ceftriaxone), with cure rates of ceftaroline $>82 \%$ [18]. Evenmore, in a retrospective integrated analysis of the FOCUS trials clinical response rates associated with the most common pathogens were numerically higher for ceftaroline compared to ceftriaxone ( $84 \%$ vs. $78 \%$, respectively) [19].

Ceftaroline is usually well tolerated, and in clinical trials only about $3 \%$ of subjects discontinued therapy due to adverse effects, most commonly due to allergic reactions. The most common adverse effects were rash, diarrhea, diarrhea, headache, hypokalaemia, insomnia and phlebitis [18].

It has been the only FDA (10/2010) approved cephalosporin for treatment of skin and soft tissue infections (cSSSIs) and CAP, in an i.v. dosis of $600 \mathrm{mg} / 12 \mathrm{~h}$.

The positive attributes of ceftaroline with respect to antimicrobial stewardship programs are: the low potential for resistance development and the favorable safety and tolerability profile in clinical trials.

Its limitations are the dosing regimen: three times daily for invasive infections (no data available for continuous infusion), and the absence of an oral formulation.

Table 1

New antimicrobials against Gram $(+)$ respiratory infections.

\begin{tabular}{|c|c|c|c|c|}
\hline Compound & Formulation & Clinical indication & Stage of development & Side effects \\
\hline Telavancin & Lipoglycopeptide & $\begin{array}{l}\text { cSSSIs in USA } \\
\text { HAP in Europe }\end{array}$ & Approved & $\begin{array}{l}\text { Taste disturbance, foamy urine, renal } \\
\text { impairment }\end{array}$ \\
\hline Ceftaroline & Cefalosporin & cSSSIs and CAP & Approved by FDA and EMA & $\begin{array}{l}\text { Hypersensitivity reactions, Clostridium } \\
\text { difficile-associated Diarrhea, }\end{array}$ \\
\hline Ceftobiprole & Cefalosporin & HAP, excluded VAP & Approved in Europe & Nausea, Vomiting, taste disturbance \\
\hline Omadacycline & Tetracycline & cSSSIs, CAP & Phase III cSSTI completed & Nausea, elevation of ALT levels \\
\hline Cethromycin & Ketolide & CAP & Phase III & Diarrhea, dysgeusia, headache \\
\hline Solithromycin & Ketolide & CAP & Phase III & Diarrhea, headache and nausea \\
\hline Tedizolid & oxazolidinone & cSSSIs and HAP & Phase III cSSTI \& HAP & Nausea, diarrhea, headache, vomiting \\
\hline Nemonoxacin & Quinolone & CAP & Phase III clinical trial in CAP & Headache, contact dermatitis, pruritus, rash \\
\hline
\end{tabular}

NDA: new drug application, cSSSIs: complicated skin and skin structure infection, CAP: community acquired pneumonia. 
Table 2

Chemical structures of new antibiotics.

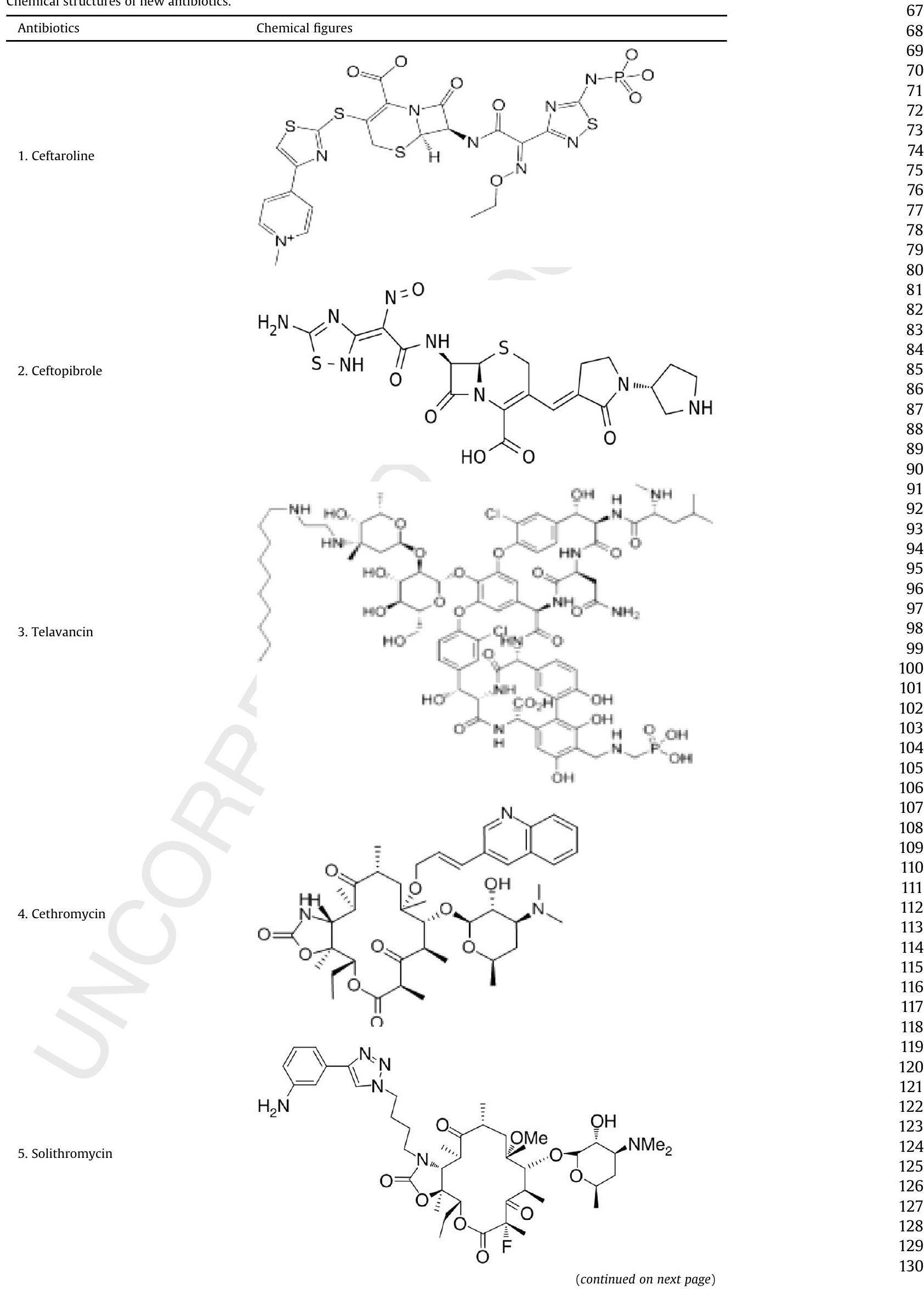


Table 2 (continued)

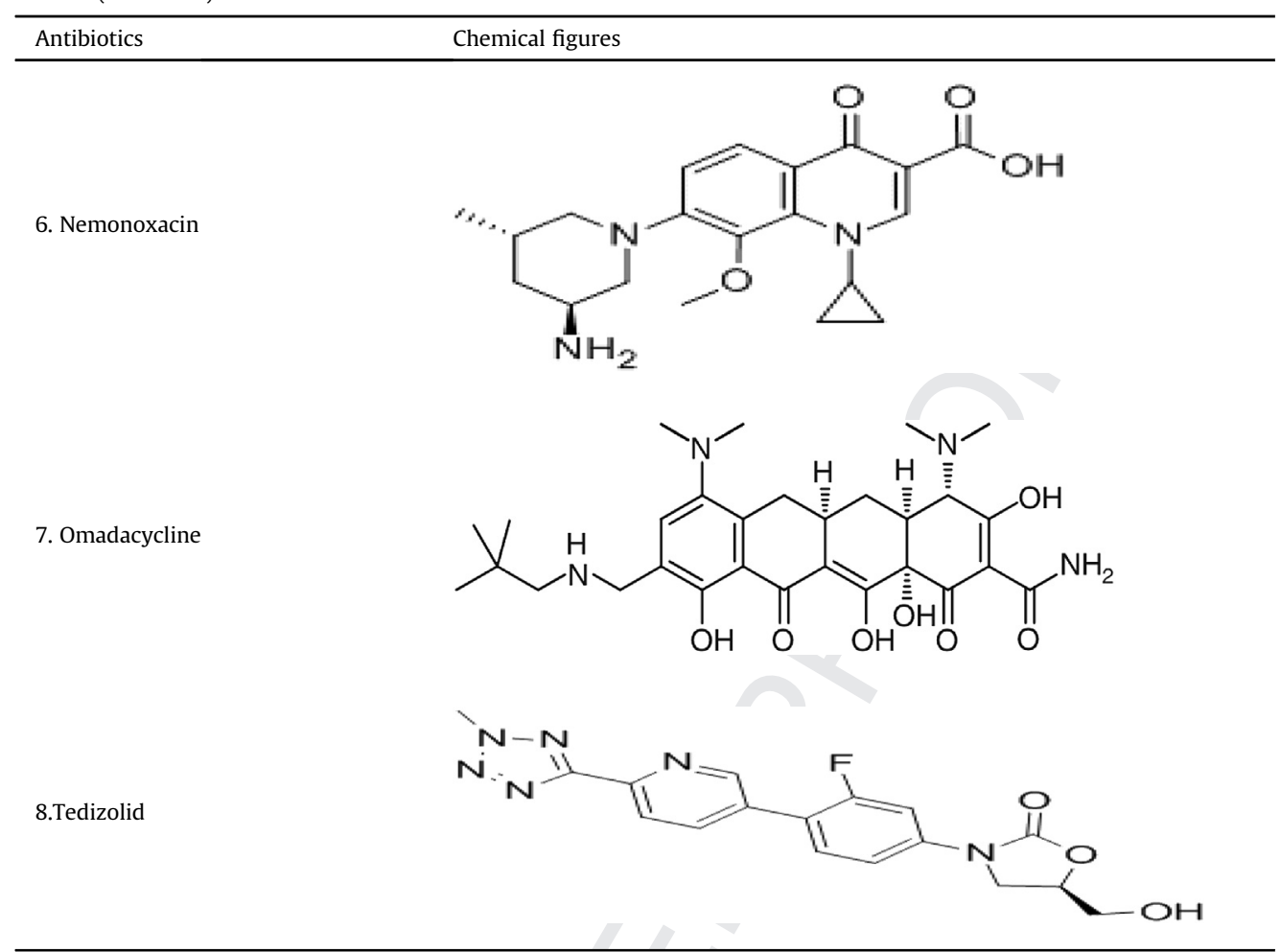

\section{Ceftopibrole}

Ceftobiprole is a new member of the pyrrolidone-3ylidenemethyl cephem series of cephalosporins. It is active against pneumococci, S. aureus-including MRSA and vancomycinintermediate S. aureus (VISA), Enterococcus faecalis and Gramnegative bacilli-especially has anti-pseudomonal activity similar to cefepime and some anaerobes [20].

Like ceftaroline has strong binding affinity to PBP-2x and PBP-2a resulting in bactericidal activity against resistant $S$. pneumoniae and Ca-MRSA.

MICs for all streptococcal species, except the penicillin-resistant Streptococcus viridans, but including penicillin-resistant S. pneumoniae, ranged from $<$ or $=0.008-2.0 \mathrm{mg} / \mathrm{L}$. Ceftobiprole is active against E. faecalis but generally not active against Enterococcus faecium. A surveillance study on S. pneumoniae called, TRUST 12, showed that ceftobiprole was the most potent cephalosporin tested against S. pneumoniae with $\mathrm{MIC}_{50}(0.015 \mu \mathrm{g} / \mathrm{mL})$ and $\mathrm{MIC}_{90}(0.5 \mu \mathrm{g} /$ $\mathrm{mL}$ ) with values two-fold lower than ceftriaxone [21].

PK/PD studies in humans demonstrate that ceftobiprole has an elimination half-life of about $3 \mathrm{~h}$ and predominantly urinary excretion.

In the phase III study published by Nicholson et al. [22], ceftobiprole $500 \mathrm{mg}$ every $8 \mathrm{~h}$ was non-inferior to ceftriaxone with or without linezolid for the treatment of CAP patients. Also, for HAP, a phase III clinical trial demonstrates the noninferiority of ceftobiprole plus placebo compared with linezolid plus ceftazidime with respect to the clinical cure (77\% for ceftobiprole and $76 \%$ for combination therapy). However, ceftobiprole was inferior in the subgroup of patients undergoing mechanical ventilation (VAP) [13].

In a more recent review about ceftobiprole [23], reported that in patients with normal PK and non-VAP, ceftobiprole is effective for the treatment of HAP in the recommended doses, but it is unlikely to achieve the desired PD targets when PK parameters are altered in VAP (e.g., increased Vd and Cl). That's why it has been approved in Europe for HAP, but not VAP.

It is well tolerated with frequently reported adverse effects included taste disturbances, nausea and vomiting.

\section{Telavancin}

Telavancin is a vancomycin-derived lipoglycopeptide which is characterized by a broad spectrum of microbiologic activity against Gram-positive bacteria, such as $S$. pneumoniae independent of penicillin susceptibility and staphylococci (including MRSA, hVISA and VISA strains) [24].

Its antibacterial effects are proposed to be achieved through a dual mechanism of action, i.e. inhibition of cell wall peptidoglycan synthesis and depolarization of the cell membrane, resulting in disruption of the functional integrity [25].

The excellent activity of telavancin against Staphylococcus spp. represents the main characteristic of this compound. MIC values $\left(\mathrm{MIC}_{90}\right)$ of tested strains between 0.25 and $1 \mathrm{mg} / \mathrm{L}$ have been reported in an over 4500 isolates of MSSA and MRSA worldwide [26]. Against $S$. aureus with reduced susceptibility to glycopeptides, telavancin retains some activity but with elevated MICs [27]. In several studies, telavancin MICs for MRSA ranged from two to eight times lower than those observed for vancomycin, teicoplanin and linezolid.

It is highly protein bound estimated at $93 \%$ and has a long halflife (up to $9 \mathrm{~h}$ ), so that it can be given once a day. Telavancin excreted mainly via the kidneys, so the dose of the drug needs adjustment for patients with moderate renal insufficiency.

Telavancin distributed into lung tissue with epithelial lining fluid concentrations of approximately two- to eightfold above the $\mathrm{MIC}_{90}$ of $S$. aureus for $24 \mathrm{~h}$. Post antibiotic effect of $4-6 \mathrm{~h}$ were 
demonstrated against a variety of Gram-positive bacteria, including MSSA and MRSA [28].

Telavancin has been also evaluated in two studies (ATTAIN 1-2) in the treatment of HAP due to Gram-positive cocci, particularly MRSA. In these studies telavancin was noninferior to vancomycin on the basis of clinical response in the treatment of HAP, especially clinical cure: $82 \%$ telavancin. vs. $74 \%$ for vancomycin. But in patients with pre-existing moderate/severe renal impairment $(\mathrm{CrCl}<50 \mathrm{~mL} / \mathrm{min})$ telavancin presented an increased mortality compare to vancomycin [29].

Responding to this result. in a post hoc analysis of data from two Phase 3 ATTAIN trials [30] excluding patients with severe renal impairment (creatinine clearance $<30 \mathrm{~mL} / \mathrm{min}$, including patients on haemodialysis) and pre-existing acute renal failure, the clinical and safety outcomes were similar in the telavancin and vancomycin treatment groups.

The ATTAIN studies also reported adverse events being gastrointestinal discomfort and taste disturbance, nausea, headache, vomiting, insomnia and foamy urine.

FDA and European Medicines Agency (EMEA) accepted telavancin for the treatment for adults with nosocomial pneumonia, including VAP known or suspected to be caused by MRSA, in patients without renal insufficiency [31].

\section{Under development antimicrobials}

\subsection{Cethromycin}

Cethromycin is a new fluoroketolide with a reported high potency against Gram-positive, Gram-negative bacteria and atypicals including mycoplasma and ureaplasma. It has also in vitro activity against penicillin- and macrolide-resistant Gram-positive organisms, possibly due to a higher affinity for the target site on the ribosomal unit [32].

The SENTRY Antimicrobial Surveillance Program platform has shown that cethromycin was highly active in vitro against all Grampositive organisms $\left(\mathrm{MIC}_{50}, 0.015 \mu \mathrm{g} / \mathrm{mL}\right)$ as compared with telithromycin ( $\mathrm{MIC}_{50}, 0.06 \mu \mathrm{g} / \mathrm{mL}$ ), clarithromycin ( $\mathrm{MIC}_{50}, 0.12 \mu \mathrm{g} / \mathrm{mL}$ ), and erythromycin $\left(\mathrm{MIC}_{50}, 0.25 \mu \mathrm{g} / \mathrm{mL}\right)$ [33]. Cethromycin had excellent activity against $S$. pneumoniae, including isolates harboring mef (A) ( $\left.\mathrm{MIC}_{90}, 0.06 \mu \mathrm{g} / \mathrm{mL}\right)$, or erm (B) ( $\mathrm{MIC}_{90}, 0.25 \mu \mathrm{g} /$ $\mathrm{mL}$ ), penicillin-resistant pneumococci $\left(\mathrm{MIC}_{90}, 0.12 \mu \mathrm{g} / \mathrm{mL}\right)$, and serotype $19 \mathrm{~A}$ pneumococci ( $\mathrm{MIC}_{90}, 0.12 \mu \mathrm{g} / \mathrm{mL}$ ) [34].

Cethromycin distributes well into pulmonary compartments and at concentrations higher than plasma, with a daily dose of 150 or $300 \mathrm{mg}$ yielding a Cmax in the ELF of 0.94 or $2.75 \mu \mathrm{g} / \mathrm{mL}$ in two to four hours and AUC0-24values of 11.4 and $24.15 \mu \mathrm{g} \mathrm{h} / \mathrm{mL}$ [35].

There are two comparative CAP studies of cethromycin $300 \mathrm{mg}$ per day to clarithromycin $250 \mathrm{mg}$ twice a day that demonstrating the noninferiority of cethromycin in terms of clinical cure and radiographic improvement [36], in low severity CAP patients. The reported most common adverse events were diarrhea, nausea, dysgeusia and headache.

Cethromycin completed Phase III clinical trials for the treatment of CAP and was deemed to be safe according to the FDA Advisory Committee, after dosing more than 5000 patients in 53 clinical studies for patients with mild or moderate CAP [37]. But more trials will be needed with more severe CAP patients before approval can be granted.

\section{Solithromycin}

Solithromycin is a novel fluoroketolide with high potency against Gram-positive and Gram-negative bacteria commonly associated with community-LRTIs. Against strains with defined susceptibilities to erythromycin, clindamycin and telithromycin, solithromycin showed potent inhibition against all combinations $\left(\mathrm{MIC}_{50}=0.06 \mu \mathrm{g} / \mathrm{mL}\right)$ except those with non-susceptibility to telithromycin $(>2 \mu \mathrm{g} / \mathrm{mL})\left(\mathrm{MIC}_{50}>16 \mu \mathrm{g} / \mathrm{mL}\right)$ [38].

Solithromycin has in vitro potency against the major CABP pathogens, S. pneumoniae ( $\mathrm{MIC}_{90}, 0.03 \mathrm{~g} / \mathrm{mL}$ ), Haemophilus influenzae ( $\mathrm{MIC}_{90}, 2 \mu \mathrm{g} / \mathrm{mL}$ ), Moraxella catarrhalis ( $\mathrm{MIC}_{90}, 0.06 \mu \mathrm{g} / \mathrm{mL}$ ), and MSSA; $\mathrm{MIC}_{90}, 0.06 \mu \mathrm{g} / \mathrm{mL}$ ), including intracellular pathogens Legionella pneumophila ( $\mathrm{MIC}_{90}, 0.016 \mu \mathrm{g} / \mathrm{mL}$ ), Chlamydia pneumoniae $\left(\mathrm{MIC}_{90}, 0.25 \mu \mathrm{g} / \mathrm{mL}\right.$ ), and Mycoplasma pneumoniae ( $\mathrm{MIC}_{90}$, $0.000125 \mu \mathrm{g} / \mathrm{mL})$.

The half-life increased with dose and averaged $5.1-6.5 \mathrm{~h}$ for the 400-800 mg dose. A phase 1 study to determine the ELF and alveolar macrophage (AM) levels of solithromycin after five days of oral dosing of $400 \mathrm{mg} \mathrm{q} 24 \mathrm{~h}$ found the exposure (AUCO-24) of solithromycin in ELF and AM was $>8$ times and $\geq 180$ times higher than the total plasma exposure, respectively [39].

A completed Phase 2 study showed comparable efficacy of solithromycin $800 \mathrm{mg}$ on day 1 followed by $400 \mathrm{mg}$ on days $2-5$ to levofloxacin $750 \mathrm{mg}$ once daily in adults with PSI-II to PSI-IV CABP [40]. A new global Phase 3 trial of solithromycin in patients with CABP includes a double-blind, placebo-controlled, multicenter study enrolling $~ 800$ CAP patients and randomize them to either oral solithromycin, an $800 \mathrm{mg}$ loading dose followed by $400 \mathrm{mg}$ once daily for 5 days or once-daily oral moxifloxacin $400 \mathrm{mg}$ for 7 days is taken place now and the results are expected with interest [41].

Across all the studies, the most common adverse events of the drug were diarrhea (13\%), headache (13\%) and nausea (10\%), most of which were mild.

\section{Nemonoxacin}

Nemonoxacin is a novel non-fluorinated quinolone that is a broad-spectrum, once daily oral therapy for cSSSI and CAP.

Nemonoxacin displayed greater activity than the fluoroquinolones (levofloxacin) against MSSA, MSSE, MRSE, $S$. pneumoniae, and E. faecalis. Interestingly, nemonoxacin maintained better activity against CA-MRSA than against HA-MRSA. It has also demonstrated a potent antibacterial activity against ciprofloxacin-resistant MRSA, methicillin- and levofloxacinresistant Staphylococcus capitis, penicillin and levofloxacinresistant S. pneumoniae and VRE $[42,43]$.

Oral nemonoxacin (750 mg or $500 \mathrm{mg}$ ) administered once daily for seven days showed similar clinical and bacteriological response as levofloxacin (500 mg once daily) in the therapy of CAP [44]. Usual adverse events were diarrhea, dizziness and headache.

It has currently completed one FDA Phase 2 trial specifically for diabetic foot infections and 2 new Phase 3 clinical comparative trials with levofloxacin in patients with CAP [45].

\section{Omadacycline}

Omadacycline, an aminomethylcycline, is a semisynthetic derivative of minocycline that has in vitro potency against Grampositive and Gram-negative bacteria and atypicals ( $L$. pneumophila) causing ASSSIs and CAP.

Like tigecycline, potent activity was observed in vitro against resistant Gram-positive bacteria, with $\mathrm{MIC}_{90}$ values $\leq 0.5 \mathrm{mg} / \mathrm{ml}$. The omadacycline $\mathrm{MIC}_{90}$ s for MRSA, VRE, and beta-hemolytic streptococci were $1.0 \mu \mathrm{g} / \mathrm{mL}, 0.25 \mu \mathrm{g} / \mathrm{mL}$, and $0.5 \mu \mathrm{g} / \mathrm{mL}$, respectively, and for PRSP and H. influenzae were $0.25 \mu \mathrm{g} / \mathrm{mL}$ and $2.0 \mu \mathrm{g} /$ $\mathrm{mL}$, respectively. Omadacycline was active against organisms demonstrating the two major mechanisms of resistance, ribosomal protection and active tetracycline efflux [46]. 
The in vitro activity of omadacycline was also superior to doxycycline, minocycline, clindamycin, linezolid, or vancomycin against enterococcus, including vancomycin-resistant $E$. faecalis or E. faecium, and S. pneumoniae strains including penicillin- and multiresistant strains.

Omadacycline is metabolically stable and has demonstrated low protein binding across all concentrations and species tested [47] and is orally absorbed.

In a Phase 3 study of patients with complicated skin and soft tissue infections (cSSSI), oral and i.v. omadacycline was well tolerated, with efficacy demonstrating comparability with linezolid [48].

The most frequently reported AEs in the IV studies were cannula site reactions and elevated ALT levels ranging from 1.9 to $3.8 \times$ the upper limit of normal (ULN) in 300-600 $\mathrm{mg}$ IV doses. Nausea (25\%) was observed after oral administration only. All AEs were mild in intensity and resolved by the end of study $[48,49]$.

On January 2013, FDA has designed omadacycline as a Qualified Infectious Disease Product (QIDP) for both IV and oral formulations in the treatment of acute bacterial skin and skin structure infections and bacterial CAP.

\section{Oxazolidones}

Tedizolid and radezolid are two new oxazolidinones that are currently under development; both retain activity against MRSA strains that are resistant to linezolid [50].

Tedizolid phosphate, is a new oxazolidinone prodrug that is transformed in the serum into the active drug torezolid. Tedizolid acts by inhibiting protein synthesis and has broad activity against Gram (+) pathogens. The methyl tetrazole D-ring system pick up (Table 2) additional binding site interactions with the ribosome and thus confer increased potency, relative to that of linezolid.

Tedizolid is four to eightfold more active in vitro than linezolid against staphylococci, streptococci and enterococci. Close to $80 \%$ of linezolid resistant strains were inhibited by tedizolid at a concentration of $\leq 4 \mu \mathrm{g} / \mathrm{mL}[51]$.

It is highly potent prodrug with good drug properties, solubility of $\geq 130 \mathrm{mg} / \mathrm{mL}$ in aqueous solutions of $\mathrm{pH} \geq 5.0$, and $91.7 \%$ oral bioavailability. The elimination half-life $(8-11 \mathrm{~h})$ and volume of distribution for tedizolid were nearly double the values for linezolid. It has an intravenous/oral step-down formulation as well as a 6 day oral dosing regimen [52].

Unlike linezolid, it demonstrate 10-fold accumulation in human macrophages as well intracellular killing of phagocytosed S. aureus, Listeria monocytogenes and L. pneumophila, which contributes to its improved pharmacodynamic properties. Lemaire et al. demonstrated that tedizolid penetrates into macrophages cultured in vitro and kills intracellular staphylococci better than linezolid, but that killing is directly related to MIC differential [53].

It shows an improved safety profile that includes decreased haematological effects at the therapeutic dose [54] and the absence of a pressor effect in response to tyramine challenge in an animal model. Unlike linezolid, tedizolid does not inhibit monoamine oxidase in vivo, therefore interactions with adrenergic, dopaminergic, and serotonergic drugs are not to be expected.

In a double-blind Phase 2 clinical study, patients with cSSSIs (a vast majority had S. aureus and more than $80 \%$ had MRSA infection) were given tedizolid once a day oral doses of 200,300 or $400 \mathrm{mg}$ for 5-7 days. Clinical cure rates in excess of 95\% were achieved for MRSA as well as MSSA infections in all three dosage groups [51]. The side effects reported were nausea, stomach discomfort, diarrhea, headache, and dysgeusia.
In December 2013, the FDA designated tedizolid as a Qualified Infectious Disease Product (QIDP), for its potential indication in ABSSSI.

A Phase 3 comparative trial is currently recruiting patients with VAP and Gram (+) bacteremia to compare tedizolide $200 \mathrm{mg}$ IV once daily for 7 days to linezolid $600 \mathrm{mg}$ every $12 \mathrm{~h}$ [55].

\section{Discussion}

Whereas resistant Gram-negative bacteria were a major concern in previous years, over the last few years a dramatic increase in the resistance of Gram-positive bacteria has occurred, these have included MDR staphylococci, penicillin resistant S. pneumoniae, and VRE.

Regarding this event we have presented some of the exciting and noteworthy ongoing developments in the field of antibacterials against Gram $(+)$ respiratory pathogens. Our opinion for their clinical use depends according the site of care:

\subsection{CAP treatment}

The major problem in the management of CAP is the inability to determine the aetiological pathogen. Therefore, it is necessary to carefully consider the various risk factors and epidemiological circumstances and initiate empirical treatment with antibiotics able to effectively treat the most likely pathogens causing the infection.

Among the above mentioned new antibiotics approved or not most appropriate for outpatient treatment will be the two ketolides: cethromycin, solithromycin and the quinolone nemonoxacin. They have oral formulations and are active against the more common respiratory pathogens. The new tetracycline omadacycline needs more studies to evaluate its clinical use.

After the hospital admission we have to think Gram (-) pathogens, so we can use ceftaroline and ceftobiprole but not as monotherapy, because there are not active against atypicals.

\subsection{HAP treatment}

One of the main challenges in the management of HAP is to overcome the resistance issues. The patients with HAP and especially with VAP, they will have more often a diagnosis, so we can select an intravenous antibiotic with a specific spectrum (f.e. antiMRSA), as telavancin or tedizolid.

For the Gram (-) microorganisms the dosis of ceftobiprole is not fixed yet and if the etiologic miccroorganism is $P$. aeruginosa we can choose an antipseudomonal b-lactam or a quinolone. We recommend that companies should, in parallel to the efficacy, develop easy ways to measure blood levels of the new antibiotic especially in these antimicrobial targeting resistant microorganisms.

Apart from the above new antibiotics we need a strategy that promotes research, with more trials regarding PK/PD and safety, into new as well as known but unutilized compounds, allows efficient use, reduces unnecessary overuse, and limits the spread of antibiotic resistance.

\section{References}

[1] Blasi F, Mantero M, Santus P, et al. Understanding the burden of pneumococcal disease in adults. Clin Microbiol Infect 2012;18:1-8.

[2] Lim SS, Vos T, Flaxman AD, et al. A comparative risk assessment of burden of disease and injury attributable to 67 risk factors and risk factor clusters in 21 regions, 1990-2010: a systematic analysis for the Global Burden of Disease Study 2010. Lancet 2013;380:2224-60.

[3] Watson R. Multidrug resistance responsible for half of deaths from healthcare associated infections in Europe. BMJ 2008 Jun 7;336(7656):1266-7.

[4] Welte T, Torres A, Nathwani D. Clinical and economic burden of communityacquired pneumonia among adults in Europe. Thorax 2012;67:71-9. 
[5] Pfaller MA, Farrell DJ, Sader HS, Jones RN. AWARE Ceftaroline Surveillance Program (2008-2010): trends in resistance patterns among Streptococcus pneumoniae, Haemophilus influenzae, and Moraxella catarrhalis in the United States. Clin Infect Dis 2012;55(Suppl. 3):S187-93.

[6] Lynch III JP, Zhanel GG. Streptococcus pneumoniae: epidemiology and risk factors, evolution of antimicrobial resistance, and impact of vaccines. Curr Opin Pulm Med 2010;16:217-25.

[7] Song JH, Chang HH, Suh JY, et al. ANSORP Study Group. Macrolide resistance and genotypic characterization of Streptococcus pneumoniae in Asian countries: a study of the Asian Network for Surveillance of Resistant Pathogens (ANSORP). J Antimicrob Chemother 2004;53:457-63.

[8] Doern GV, Richter SS, Miller A, et al. Antimicrobial resistance among Streptococcus pneumoniae in the United States: have we begun to turn the corne on resistance to certain antimicrobial classes? Clin Infect Dis 2005;41:139-48

[9] Klein E, Smith DL, Laxminarayan R. Hospitalizations and deaths caused by methicillin-resistant Staphylococcus aureus, United States, 1999-2005. Emerg Infect Dis 2007;13:1840-6.

[10] Koulenti D, Lisboa T, Brun-Buisson C, et al. Spectrum of practice in the diagnosis of nosocomial pneumonia in patients requiring mechanical ventilation in European intensive care units. Crit Care Med 2009;37:2360-8.

[11] Rello J, Torres A, Ricart M, et al. Ventilator-associated pneumonia by Staphylococcus aureus: comparison of methicillin-resistant and methicillin-sensitive episodes. Am J Respir Crit Care Med 1994;150:1545-9.

[12] Bouza E, Giannella M, Bunsow E, et al. Ventilator-associated pneumonia due to methicillin-resistant Staphylococcus aureus: risk factors and outcome in a large general hospital. J Hosp Infect 2012;80:150-5.

[13] Appelbaum Peter C. 2012 and beyond: potential for the start of a second preantibiotic era? J Antimicrob Chemother 2012 Sep;67(9):2062-8.

[14] Drusano GL. What are the properties that make an antibiotic acceptable for therapy of community-acquired pneumonia? J Antimicrob Chemother 2011 Apr;66(Suppl. 3):iii61-7.

[15] Sader HS, Fritsche TR, Kaniga K, Ge Y, Jones RN. Antimicrobial activity and spectrum of PPI-0903M (T-91825), a novel cephalosporin, tested against worldwide collection of clinical strains. Antimicrob Agents Chemother 2005;49:3501-12.

[16] Mushtaq S, Warner M, Ge Y, Kaniga K, Livermore DM. In vitro activity of ceftaroline (PPI-0903M, T-91825) against bacteria with defined resistance mechanisms and phenotypes. J Antimicrob Chemother 2007 Aug;60(2): 300-11.

[17] Neuer e, Ritchie D, Micek S. New antibiotics for healthcare-associated pneumonia. Semin Resp Crit Care Med 2009;30:92-101.

[18] Low DE, File Jr TM, Eckburg PB, et al. FOCUS 2 investigators. FOCUS 2: a randomized, double-blinded, multicentre, phase III trial of the efficacy and safety of ceftaroline fosamil versus ceftriaxone in community-acquired pneumonia. J Antimicrob Chemother 2011 Apr;66(Suppl. 3):iii33-44.

[19] File Jr TM, Low DE, Eckburg PB, Talbot GH, Friedland HD, Lee J, et al. Integrated analysis of FOCUS 1 and FOCUS 2: randomized, doubled-blinded, multicenter phase 3 trials of the efficacy and safety of ceftaroline fosamil versus ceftriaxone in patients with community-acquired pneumonia. Clin Infect Dis 2010 Dec 15;51(12):1395-405. Erratum in: Clin Infect Dis 2011 Apr 1; 52(7):967.

[20] Liapikou Adamantia, Rosales-Mayor Edmundo, Torres Antonio. Pharmacotherapy for hospital-acquired pneumonia. Expert Opin Pharmacother 2014 Apr;15(6):775-86.

[21] Davies TA, Flamm RK, Lynch AS. Activity of ceftobiprole against Streptococcus pneumoniae isolates exhibiting high-level resistance to ceftriaxone. Int Antimicrob Agents 2012;39(6):534-8

[22] Nicholson SC, Welte T, File Jr TM, Strauss RS, Michiels B, Kaul P, et al A randomised, double-blind trial comparing ceftobiprole medocaril with ceftriaxone with or without linezolid for the treatment of patients with community-acquired pneumonia requiring hospitalisation. Int J Antimicrob Agents 2012 Mar;39(3):240-6.

[23] Lagacé-Wiens PR, Rubinstein E. Pharmacokinetic and pharmacodynamics evaluation of ceftobiprole medocaril for the treatment of hospital-acquired pneumonia. Expert Opin Drug Metab Toxicol 2013 Jun;9(6):789-99.

[24] Bassetti Matteo, Merelli Maria, Temperoni Chiara, Astilean Augusta. New antibiotics for bad bugs: where are we? Ann Clin Microbiol Antimicrob 2013 Aug;28(12):22.

[25] Liapikou A, Fernandez L, Torres A. Telavancin in the treatment of nosocomial pneumonia: review of the clinical evidence. Clin Investig; 2012. Future Science.

[26] Rose WE, Fallon M, Moran JJM, Vanderlooa JP. Vancomycin tolerance in methicillin-resistant staphylococcus aureus: influence of vancomycin, daptomycin, and telavancin on differential resistance gene expression. Antimicrob Agents Chemother 2012;56(8):4422-7.

[27] Leuthner KD, Cheung CM, Rybak MJ. Comparative activity of the new lipoglycopeptide telavancin in the presence and absence of serum against 50 glycopeptide non-susceptible staphylococci and three vancomycin-resistant non-susceptible staphylococci and three vancomycin-resistant Staphylococcus aureus. J Antimicrob Chemother 2006;58:338-43.

[28] Bassetti M, Mikulska M, Righi E, Nicolini L, Viscoli C. The role of telavancin in the treatment of MRSA infections in hospital. Expert Opin Investig Drugs 2009 Apr;18(4):521-9.

[29] Rubinstein E, Lalani T, Corey GR, et al. Telavancin versus vancomycin for hospital-acquired pneumonia due to gram-positive pathogens. Clin Infect Dis 2011;52:31-40.
[30] Torres A, Rubinstein E, Corey GR, Stryjewski ME, Barriere SL. Analysis of phase 3 telavancin nosocomial pneumonia data excluding patients with severe renal impairment and acute renal failure. J Antimicrob Chemother 2014 Apr;69(4):1119-26.

[31] http://www.fda.gov/newsevents/newsroom/pressannouncements/ ucm358209.htm.

[32] Putnam SD, Castanheira M, Moet GJ, Farrell DJ, Jones RN. CEM-101, a novel fluoroketolide: antimicrobial activity against a diverse collection of gram-positive and gram-negative bacteria. Diagn Microbiol Infect Dis 2010 Apr;66(4):393-401.

[33] Jones RN, Sader HS, Flamm RK. Update of dalbavancin spectrum and potency in the USA: report from the SENTRY antimicrobial surveillance program. Diagn Microbiol Infect Dis 2013;75(3):304-7.

[34] Patel SN, Pillai DR, Pong-Porter S, et al. In vitro activity of ceftaroline, ceftobiprole and cethromycin against clinical isolates of Streptococcus pneumoniae collected from across Canada between 2003 and 2008. J Antimicrob Chemother 2009;64:659-60.

[35] Conte Jr JE, Golden JA, Kipps J, Zurlinden E. Steady-state plasma and intrapulmonary pharmacokinetics and pharmacodynamics of cethromycin. Antimicrob Agents Chemother 2004;48:3508-15.

[36] English ML, Fredericks CE, Milanesio NA, Rohowsky N, Xu ZQ, Jenta TR, et al. Cethromycin versus clarithromycin for community-acquired pneumonia: comparative efficacy and safety outcomes from two double-blinded, randomized, parallel-group, multicenter, multinational noninferiority studies. Antimicrob Agents Chemother 2012 Apr;56(4):2037-47.

[37] Call P-F. Advanced Life Sciences Receives Complete Response Letter from FDA for Restanza (Cethromycin) in Community Acquired Pneumonia. PRNewswireFirst Call, June 2, http://www.drugs.com/nda/restanza090603.html; 2009.

[38] Farrell DJ, Castanheira M, Sader HS, Jones RN, et al. The in vitro evaluation of solithromycin (CEM-101) against pathogens isolated in the United States and Europe (2009). J Infect 2010;61:476-83.

[39] Rodvold KA, Gotfried MH, Okusanya OO, et al. Intrapulmonary penetration of solithroimycin (CEM-101) in healthy adult subjects, abstr. A1-690. In: Proceedings of the 50th Annual ICAAC, September 12-15, Boston, MA; 2010.

[40] Oldach D, Clark K, Schranz J, Das A, Craft JC, Scott D, et al. Randomized, double-blind, multicenter phase 2 study comparing the efficacy and safety of oral solithromycin (CEM-101) to those of oral levofloxacin in the treatment of patients with community-acquired bacterial pneumonia. Antimicrob Agents Chemother 2013 Jun;57(6):2526-34.

[41] http://www.clinicaltrials.gov/ct2/show/NCT01968733? term=solithromycin\&rank=1.

[42] Lauderdale TL, Shiau YR, Lai JF, Chen HC, King CH. Comparative in vitro activities of nemonoxacin (TG-873870), a novel nonfluorinated quinolone, and other quinolones against clinical isolates. Antimicrob Agents Chemother 2010;54(3):1338-42.

[43] Li CR, Li Y, Li GQ, Yang XY, Zhang WX, Lou RH, et al. In vivo antibacterial activity of nemonoxacin, a novel non-fluorinated quinolone. J Antimicrob Chemother 2010;65(11):2411-5.

[44] van Rensburg DJ, Perng RP, Mitha IH, Bester AJ, Kasumba J, Wu RG, et al. Efficacy and safety of nemonoxacin versus levofloxacin for community-acquired pneumonia. Antimicrob Agents Chemother 2010 Oct;54(10):4098-106.

[45] http://www.clinicaltrials.gov/ct2/show/NCT01944774? term=nemonoxacin\&rank=7.

[46] Macone AB, Caruso BK, Leahy RG, Donatelli J, Weir S, Draper MP, et al. In vitro and in vivo antibacterial activities of omadacycline, a novel aminomethylcycline. Antimicrob Agents Chemother 2014 Feb;58(2):1127-35.

[47] Chaturvedi P, Esposito C, Koroma J, et al. In vitro assessment of plasma protein binding and metabolic stability of PTK 0796, abstr 2675, poster F-760. Abstr.. In: 43rd Intersci. Conf. Antimicrob. Agents Chemother. Washington, DC: American Society for Microbiology; 2003.

[48] Noel GJ, Draper MP, Hait H, Tanaka SK, Arbeit RD. A randomized, evaluatorblind, phase 2 study comparing the safety and efficacy of omadacycline to those of linezolid for treatment of complicated skin and skin structure infections. Antimicrob. Agents Chemother 2012:56:5650-4.

[49] Gould IM, Bal AM. New antibiotic agents in the pipeline and how they can help overcome microbial resistance. Virulence 2013;4(2):185-91.

[50] Prokocimer PP, Bien P, Deanda C, Pillar CM, Bartizal K. In vitro activity and microbiological efficacy of tedizolid (TR-700) against Gram-positive clinical isolates from a phase 2 study of oral tedizolid phosphate (TR-701) in patients with complicated skin and skin structure infections. Antimicrob Agents Chemother 2012:56:4608-13.

[51] Prokocimer P, De Anda C, Fang E, Mehra P, Das A. Tedizolid phosphate vs. linezolid for treatment of acute bacterial skin and skin structure infections: the ESTABLISH-1 randomized trial. JAMA 2013 Feb 13;309(6):559-69.

[52] Flanagan SD, Bien PA, Muñoz KA, Minassian SL, Prokocimer PG. Pharmacokinetics of tedizolid following oral administration: single and multiple dose, effect of food, and comparison of two solid forms of the prodrug. Pharmacotherapy 2014: Mar;34(3):240-50.

[53] Lemaire S, Van Bambeke F, Appelbaum PC, Tulkens PM. Cellular pharmacokinetics and intracellular activity of torezolid (TR-700): studies with human macrophage (THP-1) and endothelial (HUVEC) cell lines. J Antimicrob Chemother 2009;64:1035-43.

[54] Prokocimer P, et al. Hematological effects ofTR-701, linezolid and placebo administered for 21 days in healthy subjects, abstr. F1-2069a. In: 48th Intersci. Confer. Antimicrob. Agents Chemother., October 25-28.Washington, DC; 2008.

[55] http://www.clinicaltrials.gov/ct2/show/NCT02019420? term=tedizolid\&rank=17. 